\title{
Ultrareines Citicolin in Kombination mit Homotaurin und Vitamin E bei Normaldruckglaukom: ein Fallbericht
}

\author{
Tommaso Verdina $^{a, b}$ Nicola Passarelli ${ }^{b}$ Alessandra Carlini ${ }^{b}$ Francesca Chemello $^{c}$ \\ Rodolfo Mastropasqua $^{a}$ Gian Maria Cavallini ${ }^{a}$ \\ anstitut für Ophthalmologie, Universität Modena und Reggio Emilia, Modena, Italien; \\ ${ }^{b}$ Centro Oculistico Diagnostico Chirurgico, Florenz, Italien; ${ }^{c}$ Ophthalmologie am Department für Neurologie, \\ Neuropsychologie, Morphologie und Bewegungswissenschaft, Universität Verona, Verona, Italien
}

\section{Schlüsselwörter}

Normaldruckglaukom · Gesichtsfeld · Citicolin · Homotaurin .

Neuroprotektion

\section{Zusammenfassung}

Das Normaldruckglaukom ist nach wie vor eine therapeutische Herausforderung für Ophthalmologen, da in Abwesenheit des sonst üblichen Haupt-Behandlungsziels, des erhöhten Augeninnendrucks, keine effektiven Therapien bekannt sind. Wir berichten über die Wirksamkeit von zwei neuroprotektiven Molekülen (ultrapures Citicolin plus Homotaurin) in Kombination mit einer topischen drucksenkenden Therapie zur Behandlung des NTG in einer langfristigen Nachbeobachtung (30 Monate). Eine 38-jährige Frau kaukasischen Ursprungs ohne signifikante medizinische Vorgeschichte und mit diagnostiziertem NTG zeigte nach umfassender, 30-monatiger Anwendung von oraler Nahrungsergänzung mit ul- trareinem Citicolin 500 mg, Homotaurin 50 mg und Vitamin E einmal täglich sowie von topischer Glaukom-Medikation (Brimonidin + Brinzolamid als Tropfen zweimal täglich) signifikante Verbesserungen des Gesichtsfelds laut automatisierter 24-2-Untersuchung mit kontrollierter tonometrischer Messung, begleitet von einer Stabilisierung der retinalen Nervenfaserschicht und der Ganglionzellen laut OCT-Untersuchung sowie von der Zufriedenheit der Patientin. Diese Ergebnisse deuten darauf hin, dass ultrareines Citicolin in Kombination mit Homotaurin und Vitamin E durch einen synergetischen neuroprotektiven Effekt einen vielversprechenden Ansatz für das NTG-Management darstellen könnte.

c 2020 The Author(s)

\section{Einleitung}

Das Normaldruckglaukom (NTG; normal tension glaucoma) ist eine Form des Offenwinkelglaukoms, bei der die glaukomtypischen Veränderungen des Sehnervenkopfs und funktionellen Gesichtsfeldverlusts vorliegen, der Augeninnendruck (AID) jedoch nicht erhöht ist [1].

Seine Vorkommenshäufigkeit wird mit $30-40 \%$ aller Fälle von glaukombedingten Gesichtsfeldausfällen beziffert. Die Ätiologie ist bisher nicht abschließend geklärt, scheint jedoch multifaktoriell zu sein, und häufig ist das Glaukom vergesellschaftet mit vaskulärer Dysregulation, nächtlicher Hypotonie und Perfusionsstörungen des Sehnervs [2].

Wie das NTG zu behandeln ist, ist Gegenstand einiger Diskussion, da der erhöhte AID, der sonst das Haupt-Therapieziel ist, hier nicht vorliegt. Die Collaborative Normal Tension Glaucoma Study ergab bei Patienten, deren AID um 30\% oder mehr gesenkt wurde, eine verlangsamte Krankheitsprogression [3]. Bei Patienten, die mit topischem Brimonidin behandelt wurden, schien die Wahrscheinlichkeit von Verschlechterungen des Gesichtsfelds niedriger als bei denen, die mit Timolol behandelt wurden [4]; der Goldstandard der medikamentösen Therapie bei NTG ist jedoch 


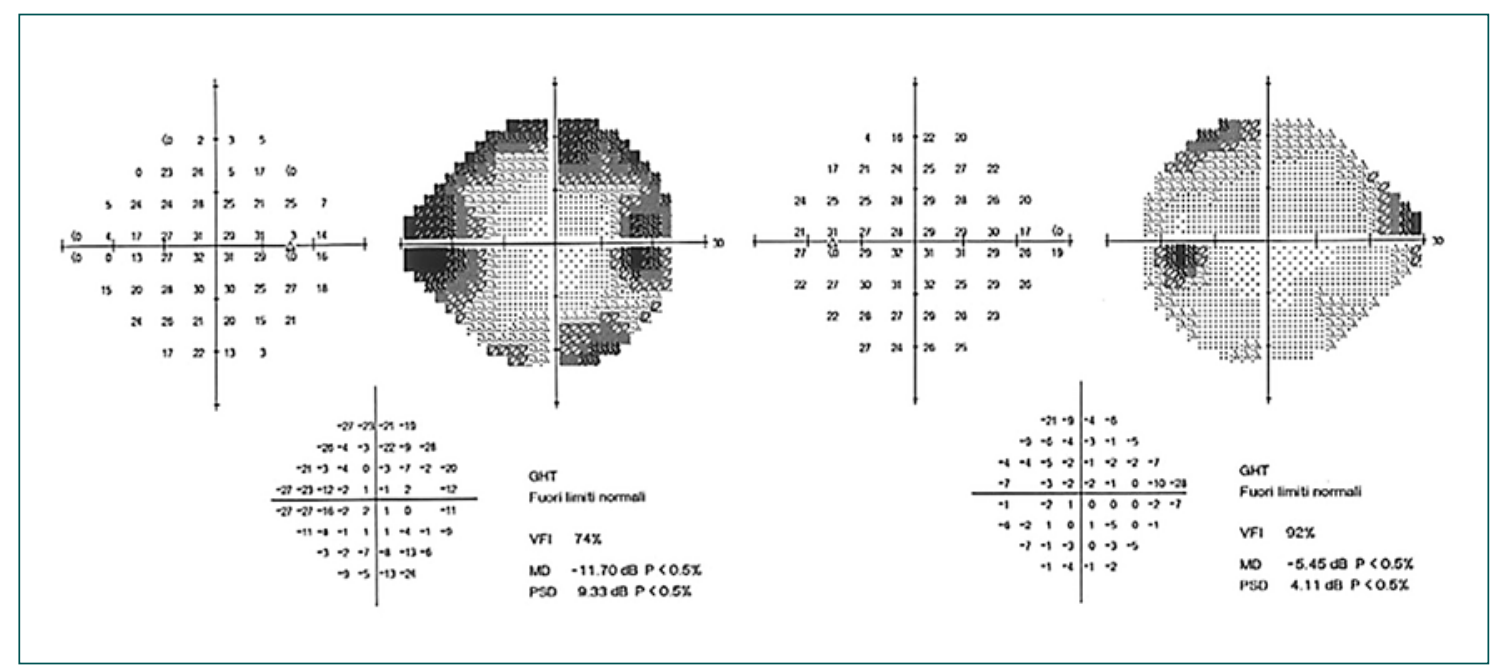

Abb. 1. Gesichtsfeld 24-2 (RA links und LA rechts im Bild) bei der Eingangsuntersuchung.

derzeit die topische Anwendung von Prostaglandin-Analoga [5, 6]. In den letzten Jahren hat sich beim Offenwinkelglaukom der Blick verstärkt auf die systemische Neuroprotektion durch spezielle Nahrungsergänzungsmittel gerichtet; beim NTG wird ihr Einsatz noch diskutiert $[7,8]$.

Ultrareines Citicolin oder Cytidin-5-diphosphochilon (CDPCholin, Cognizin ${ }^{\circledR}$ ) ist ein endogenes Molekül von zentraler Bedeutung für die Prozesse der Bildung und Wiederherstellung der Nervenzellen, die mit der Synthese der Phospholipide von Membranzellen interagieren [9]; darüberhinaus spielt das Molekül eine wichtige Rolle bei der Bildung von Acetylcholin, das als Neurotransmitter essenziell für die Impulsübermittlung im Gehirngewebe ist. Seine neuroprotektive Wirkung ist für viele verschiedene neurodegenerative Erkrankungen nachgewiesen, von Alzheimer-Demenz über Morbus Parkinson bis hin zum Glaukom [10]. Ultrareines Citicolin hat sich außerdem als leistungssteigernd für die Aufmerksamkeit bei gesunden weiblichen Erwachsenen sowie die psychomotorische Geschwindigkeit bei männlichen Jugendlichen erwiesen $[11,12]$.

Homotaurin (3-Aminopropansulfonsäure) ist ein AminosäureDerivat, das helfen kann, die Erregungsleitung im zentralen Nervensystem zu verbessern. In einer aktuellen Studie zeigte die Behandlung mit Citicolin plus Homotaurin eine positive und synergetische Wirkung auf das Zellüberleben in Retina-Kulturen zur Simulation retinaler Neurodegeneration unter experimentellen Bedingungen [13].

Vitamin E (alpha-Tocopherol) ist ein Antioxidans, das zum Zellschutz beiträgt [14].

Wir berichten hier über den Fall einer jungen Frau, der zur Behandlung eines NTG ultrareines Citicolin, Homotaurin und Vitamin E verabreicht wurden.

\section{Vorstellung des Falls}

Eine 38-jährige Frau wurde zu einer allgemeinen ophthalmologischen Untersuchung an unsere Klinik überwiesen (Eingangsun- tersuchung). Signifikante Allgemeinerkrankungen lagen nicht vor. Die Patientin klagte über häufige Migräneepisoden. Die Familienanamnese war negativ für Augenerkrankungen. Die beste korrigierte Sehschärfe betrug 20/20 mit einer Refraktion von $-0,75$ und -1,75 (170) im rechten Auge (RA) und 20/20 mit -2,00 (160) im linken (LA). Der AID betrug $14 \mathrm{mmHg}$ im RA und 12 mmHg im LA. Die Biomikroskopie des vorderen Segments war beidseitig unauffälllig. Die Öffnung des Kammerwinkels lag im normalen Bereich; alle Winkelstrukturen waren gut erkennbar. Die Ophthalmoskopie ergab beidseitig eine anomale Aushöhlung des Sehnervenkopfs (Papillenexkavation). Daher erfolgten eine Gesichtfelduntersuchung mittels Humphrey SITA Standard 24-2 (HVF; Carl Zeiss Meditech, Dublin, CA, USA) sowie eine OCTUntersuchung der retinalen Nervenfaserschicht (RNFL), Ganglionzellen (GC) und Hornhautpachymetrie (Cirrus 4000 HDOCT, Carl Zeiss Meditec, Dublin, CA, USA) (Abb. 1-3).

Die mittlere Abweichung (MD; mean deviation) betrug -11,70 im RA und $-5,45$ im LA. Falsch-Negative lagen zu $26 \%$ beim RA und $0 \%$ beim LA vor. Die mittlere RNFL betrug in beiden Augen $76 \mu \mathrm{m}$; der GC-Wert lag bei $76 \mu \mathrm{m}$ im RA und $66 \mu \mathrm{m}$ im LA. Der CDRWert (cup-to-disc ratio) betrug in beiden Augen 0,83. Die Hornhautpachymetrie ergab für das RA $512 \mu \mathrm{m}$ und für das LA $516 \mu \mathrm{m}$. Wir ordneten eine MRT-Untersuchung (Magnetresonanztomografie) der Orbita und des Enzephalons an. Eine topische Therapie mit Brimatoprost-Tropfen $0,1 \mathrm{mg} / \mathrm{ml}$ einmal täglich wurde eingeleitet.

Bei der Nachuntersuchung nach 1 Monat betrug der AID 11 $\mathrm{mmHg}$ im RA und $9 \mathrm{mmHg}$ im LA. Da jedoch eine Rötung der Konjunktiva vorlag und die Patientin über Beschwerden klagte, modifizierten wir die topische Therapie und verordneten Brimonidin + Brinzolamid als Tropfen zweimal täglich, begleitet von oraler Supplementierung mit ultrareinem Citicolin 500 mg, Homotaurin 50 mg und Vitamin E (Neuprozin Tabletten, FB Vision, 63100 Ascoli Piceno, Italien) einmal täglich für 3 Monate, gefolgt von 1 Monat Anwendungspause. Die Patientin wurde aufgefordert, diese Therapie bis auf Weiteres fortzuführen.
102

Kompass Ophthalmol 2021;7:101-105 DOI: $10.1159 / 000516350$ 


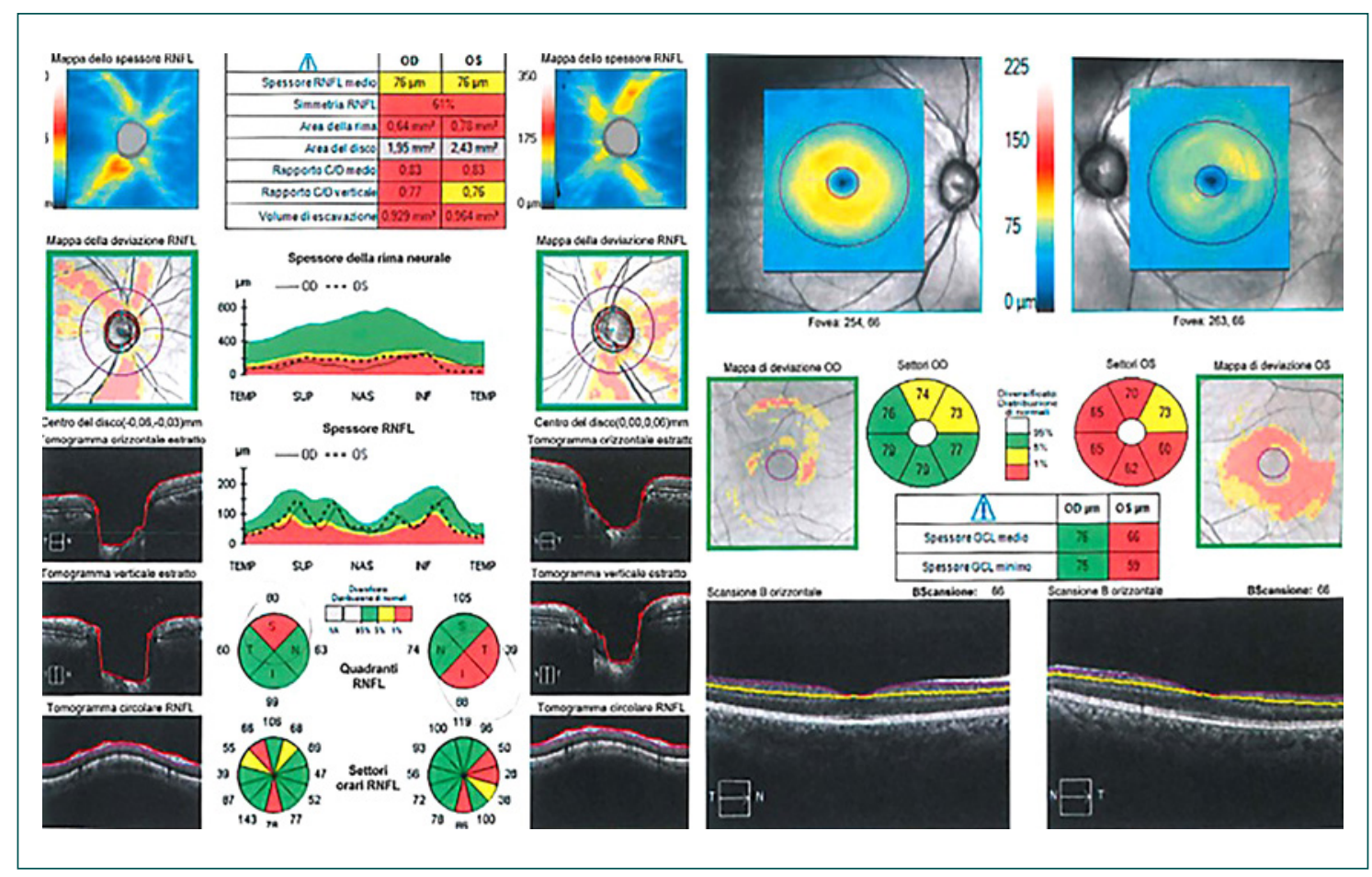

Abb. 2. OCT-Analyse (RNFL links, Ganglionzellen rechts im Bild) bei der Eingangsuntersuchung.

Abb. 3. Papille in der Retinographie bei der Eingangsuntersuchung.

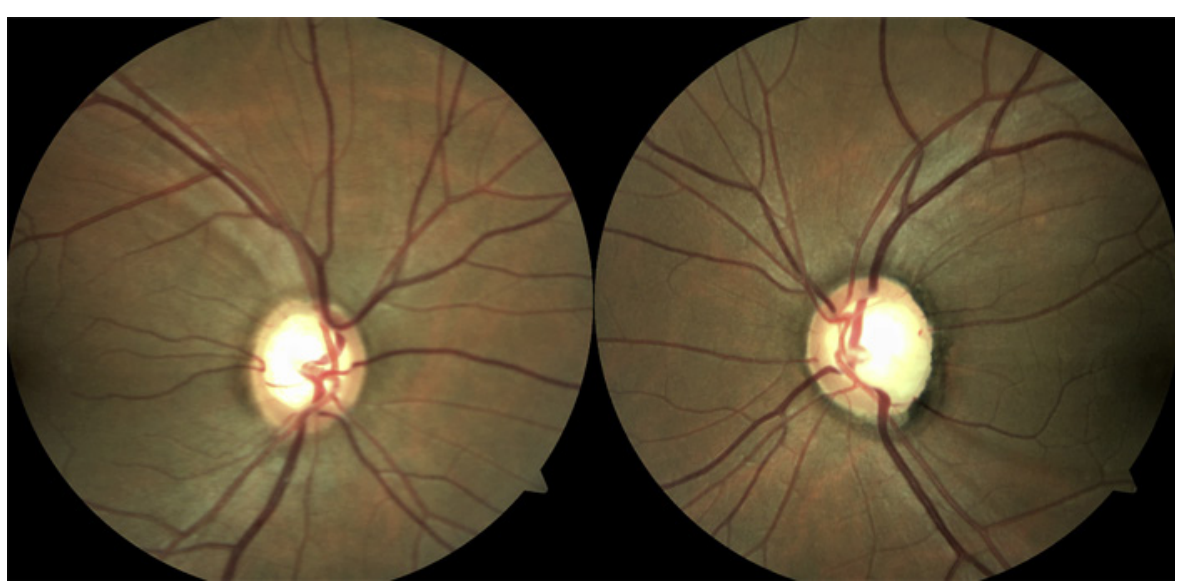

Eine erneute MRT innerhalb von 2 Wochen ergab keine wesentlichen Veränderungen.

Bei der Nachuntersuchung nach 3 Monaten hatte sich das HVFErgebnis verbessert (MD im RA von $-11,70$ auf $-7,87$; im LA von $-5,45$ auf -3,47); die Falsch-Negativ-Rate betrug beim RA $8 \%$ und beim LA 10\%. Die RNFL- und GC-Werte waren stabil. Der AID betrug $12 \mathrm{mmHg}$ im RA und $10 \mathrm{mmHg}$ im LA. Wir ordneten ein 24-h-Blutdruckmonitoring an.

Das 24-h-Blutdruckmonitoring ergab einen mittleren systolischen und diastolischen Druck von 114 bzw. $73 \mathrm{mmHg}$; in der Nacht betrugen die Mittelwerte $107 \mathrm{mmHg}$ systolisch und 56 $\mathrm{mmHg}$ diastolisch. Die Diagnose eines leicht erniedrigten Blutdrucks wurde gestellt.
Bei der Nachuntersuchung nach 8 Monaten betrug der AID beidseitig $11 \mathrm{mmHg}$. Die Therapie mit topischen Tropfen und oraler Medikation wurde fortgesetzt. Bei der Nachuntersuchung nach 10 Monaten betrug der AID $10 \mathrm{mmHg}$.

Bei der Nachuntersuchung nach 12 Monaten hatte sich das HVFErgebnis verbessert (MD im RA -6,71, im LA -1,20; Falsch-Negativ-Rate beim RA 7\% und beim LA 0\%), und die RNFL- und GC-Werte in der OCT waren stabil. Der AID betrug beidseitig 11 $\mathrm{mmHg}$. Die verordnete Therapie wurde weiter fortgeführt.

Danach erschien die Patientin aus persönlichen Gründen 1 Jahr lang nicht zur Kontrolluntersuchung.

Nach 24 Monaten kam sie erneut zur Nachuntersuchung in unsere Klinik. Sie gab an, die Therapie (oral und topisch) unverändert fortgeführt zu haben. Der AID betrug beidseitig $11 \mathrm{mmHg}$. Das 


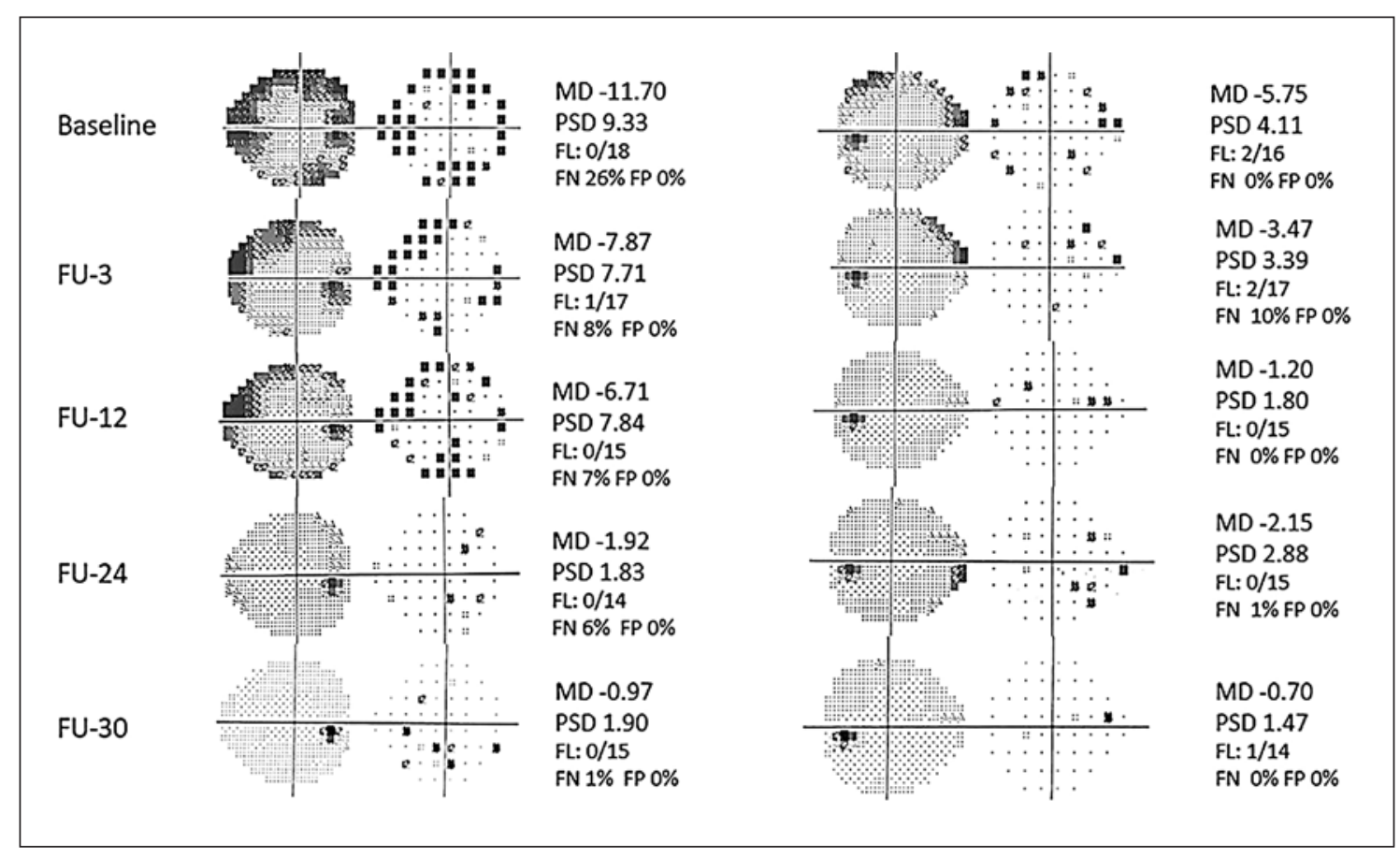

Abb. 4. Entwicklung des Gesichtsfelds in beiden Augen im Verlauf der Nachbeobachtung. MD: mean deviation; PSD: pattern standard deviation; FL: Fixationsverlust; FN: falsch negativ; FP: falsch positiv; FU-X: X-Monats-Follow-up.

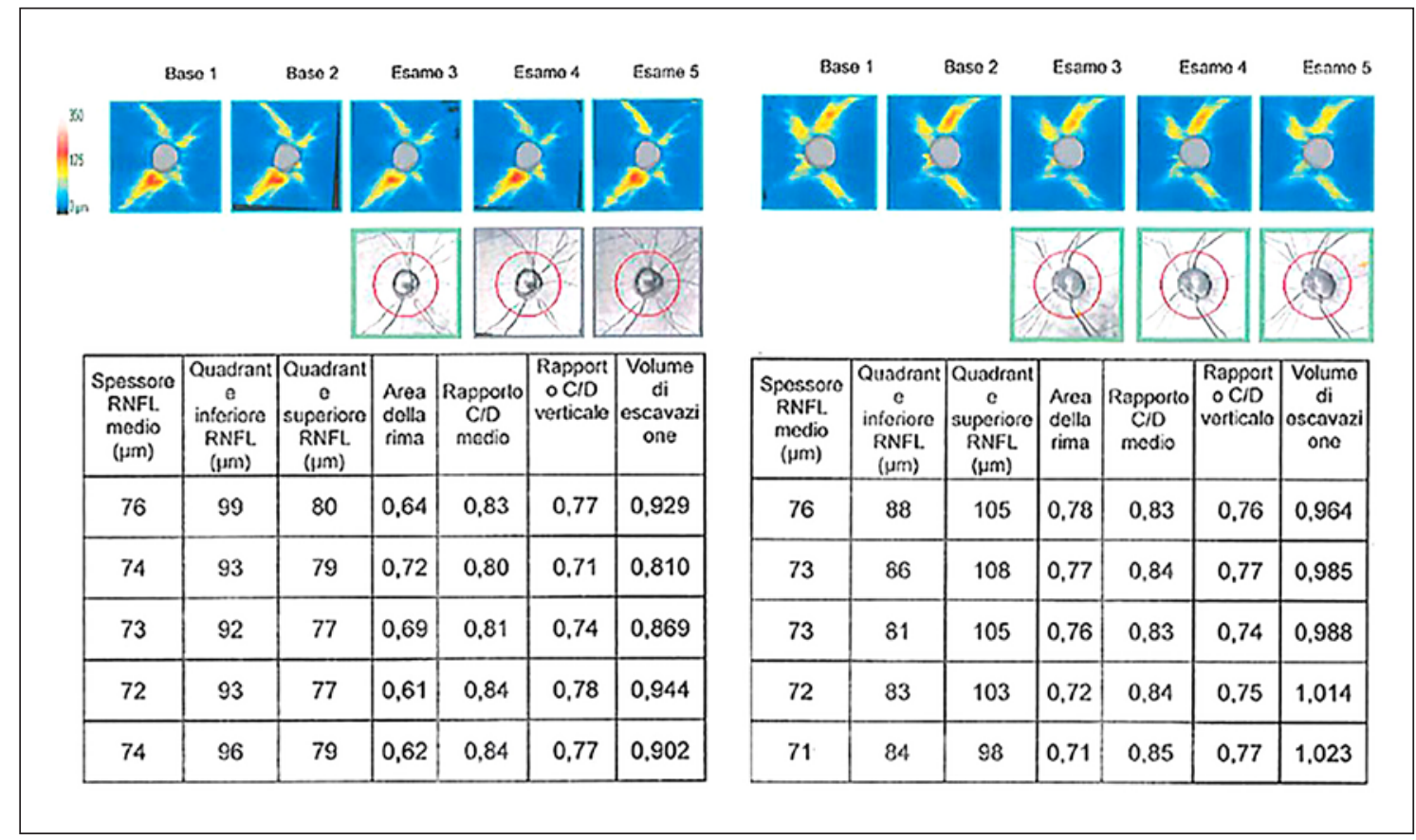

Abb. 5. Guided Progression Analysis (GPA) über den 2-jährigen Nachbeobachtungszeitraum für beide Augen. RNFL und CDR-Wert blieben stabil.

HVF-Ergebnis hatte sich weiter verbessert, besonders im rechten Auge (MD RA -1,92, LA -2,15). Die Falsch-Negativ-Rate betrug $6 \%$ beim RA und $1 \%$ beim LA; RNFL- und GC-Werte waren weiterhin stabil. Der CDR-Wert betrug beidseitig 0,84.

Bei der Nachuntersuchung nach 30 Monaten betrug der AID 11 $\mathrm{mmHg}$ im RA und $10 \mathrm{mmHg}$ im LA. Das HVF-Ergebnis zeigte weitere Verbesserung (MD RA -0,92, LA -0,70). Die FalschNegativ-Rate betrug $1 \%$ beim RA und $0 \%$ beim LA; RNFL- und GC-Werte waren weiterhin stabil.

Abbildung 4 und 5 zeigen die Ergebnisse der Nachbeobachtung. 


\section{Diskussion/Schlussfolgerungen}

In diesem Fallbericht spekulieren wir über den Nutzen des Einsatzes von Nahrungsergänzungsmitteln auf der Basis von ultrareinem Citicolin in Kombination mit Homotaurin und Vitamin E zur Behandlung des NTG.

Das Neuroenhancement der Erregungsleitung ist ein relativ neues Feld im Glaukom-Management. Ein synergetische bei mehreren Zielstrukturen ansetzendes Vorgehen mit einer Kombination aus neuroprotektiven Substanzen könnte eine vielversprechende Strategie sein, um das Fortschreiten des Glaukoms zu verlangsamen. Das NTG ist nach wie vor eine therapeutische Herausforderung für Ophthalmologen, da in Abwesenheit des sonst üblichen Haupt-Behandlungsziels, des erhöhten AID, keine effektiven Therapien bekannt sind.

Eine aktuelle Studie belegt, dass die Kombination aus Citicolin und Homotaurin synergetisch neuroprotektiv auf eine experimentelle neuroretinale Degeneration wirkte und die Apoptose primärer Retinazellen reduzierte; dies ist somit eine potenzielle neue Strategie zur Prävention und Behandlung bei neurodegenerativen Erkrankungen [10].

Darüberhinaus könnte die Hinzunahme des Antioxidans' Vitamin E durch Unterstützung des Zellschutzes das Neuroenhancement zusätzlich verbessern [14].

In unserem Bericht beschreiben wir die Verbesserung der Gesichtsfeldschädigung bei einer jungen Frau mit NTG, die durch Supplementierung mit ultrareinem Citicolin, Homotaurin und Vitamin E behandelt wurde, über einen Nachbeobachtungszeitraum von 30 Monaten. Im gesamten Nachbeobachtungszeitraum traten keine Veränderungen der OCT-Parameter ein; dies könnte als positiver Effekt im Sinne einer Stabilisierung der RNFL und GC unter der Therapie gewertet werden.

Der Bericht weist einige Beschränkungen auf, die zu beachten sind. Zum ersten ist die HVF-Untersuchung ein subjektiv geprägter psychisch-physischer Test, der durch viele Faktoren beeinflusst werden kann (z.B. wenn der Patient müde oder besorgt ist, die Raumausleuchtung leicht variiert etc.). Eine elektrophysiologische Untersuchung könnte mit objektiveren Daten zuverlässiger Aufschluss geben. Ebenfalls nicht auszuschließen ist ein Lerneffekt bei der HVF-Performance bei der jungen Patientin, insbesondere bei der ersten Untersuchung des RA zu Behandlungsbeginn. Und schließlich könnte die gleichzeitige Anwendung von topischen Brimonidin-Tropfen einen zusätzlichen neuroprotektiven Effekt ausgeübt haben, der synergetisch wirkte.

Zusammenfassend deuten unsere Ergebnisse darauf hin, dass die Anwendung von ultrareinem Citicolin in Kombination mit Homotaurin und Vitamin E durch einen synergetischen neuroprotektiven Effekt einen vielversprechenden Ansatz für das NTGManagement darstellen könnte. Kontrollierte klinische Studien sind in der Zukunft erforderlich, um diese Daten zu bestätigen.

\section{Ethikerklärung}

Diese Forschungsarbeit wurde ethisch in Übereinstimmung mit der Erklärung des Weltärztebundes von Helsinki durchgeführt. Die Patientin hat nach Aufklärung ihre Einwilligung zur Veröffentlichung ihres Falles (einschließlich der Bilder) erteilt.

\section{Disclosure Statement}

Die Autoren haben keine Interessenkonflikte offenzulegen.

\section{Finanzierungsquellen}

Es wurden keine Finanzmittel entgegengenommen.

\section{Beiträge der einzelnen Autoren}

T.V. war an Manuskriptkonzeption, Design und Datensammlung beteiligt. A.C. trug zur Datenerhebung bei. T.V. und N.P. waren an der Dateninterpretation und dem Verfassen des Entwurfs und Artikels beteiligt. F.C., R.M. und G.M.C. trugen zur kritischen Prüfung des Artikels bei. Alle Autoren genehmigten die finale Fassung des Manuskripts für die Veröffentlichung.

\section{Literatur}

Die Literatur ist unter www.karger.com/doi/10.1159/000516350 abrufbar. 